\title{
Voltage-Controlled Ferroelectric Lens Phased Arrays
}

\author{
Jaganmohan B. L. Rao, Senior Member, IEEE, Dharmesh P. Patel, Member, IEEE, \\ and Vladimir Krichevsky, Member, IEEE
}

\begin{abstract}
A new concept for phased arrays is proposed using a voltage-controlled ferroelectric lens. The ferroelectric lens concept uniquely incorporates bulk phase shifting-the array does not contain individual phase shifters-using ferroelectric material. This will reduce the number of phase shifters from $(n \times m)$ to $(n+m)$, where $n$ is the number of columns and $m$ is the number of rows in a phased array. The number of phase shifter drivers and phase shifter controls is also significantly reduced by using row-column beam steering. Thus, the ferroelectric lens concept can potentially lead to low-cost phased arrays. This paper presents the ferroelectric lens concept, theoretical analysis and design, and experimental results. The results indicate that the ferroelectric lens concept is viable and sound. Various phasedarray configurations using ferroelectric lens are included. A brief discussion on ferroelectric materials is included along with information on a U.S. Department of Defense program to improve ferroelectric materials.
\end{abstract}

Index Terms-Bulk phase shifting, ferroelectric materials/devices, lens antennas, phased arrays.

\section{INTRODUCTION}

$\mathbf{P}$ HASED-ARRAY antennas can steer transmitted and received signals without mechanically rotating the antenna. Each radiating element of a phased array is normally connected to a phase shifter and a driver, which determine the phase of the signal at each element to form a beam at the desired angle. The most commonly used phase shifters are ferrite and diode phase shifters. Phase shifters using ferroelectric materials have been proposed [1], [2]. The cost of a phased array mainly depends on the cost of phase shifters and drivers. A typical array may have several thousand elements and that many phase shifters and drivers; hence, it is very expensive. Therefore, reducing the cost and complexity of the phase shifters, drivers, and controls is an important consideration in the design of phased arrays. The phased array reported here uniquely incorporates bulk phase shifting-the array does not contain individual phase shifters-using ferroelectric material. This will reduce the number of phase shifters from $(n \times m)$ to $(n+m)$, where $n$ is the number of columns and $m$ is the number of rows in a phased array. The number of phase shifter drivers and phase shifter controls is also significantly reduced by using row-column beam steering. Bulk phase shifting using diodes has been proposed [3] and reasonably developed [4] in the radant lens. The lens described here uses voltage-controlled ferroelectric, which introduces analog phase shift rather than digital phase shift as in the radant lens. The ferroelectric lens

Manuscript received May 29, 1997; revised June 3, 1998. This work was supported by the Office of Naval Research Code ONR 31.

The authors are with the Radar Division, Naval Research Laboratory, Washington, DC 20375 USA.

Publisher Item Identifier S 0018-926X(99)04445-2.

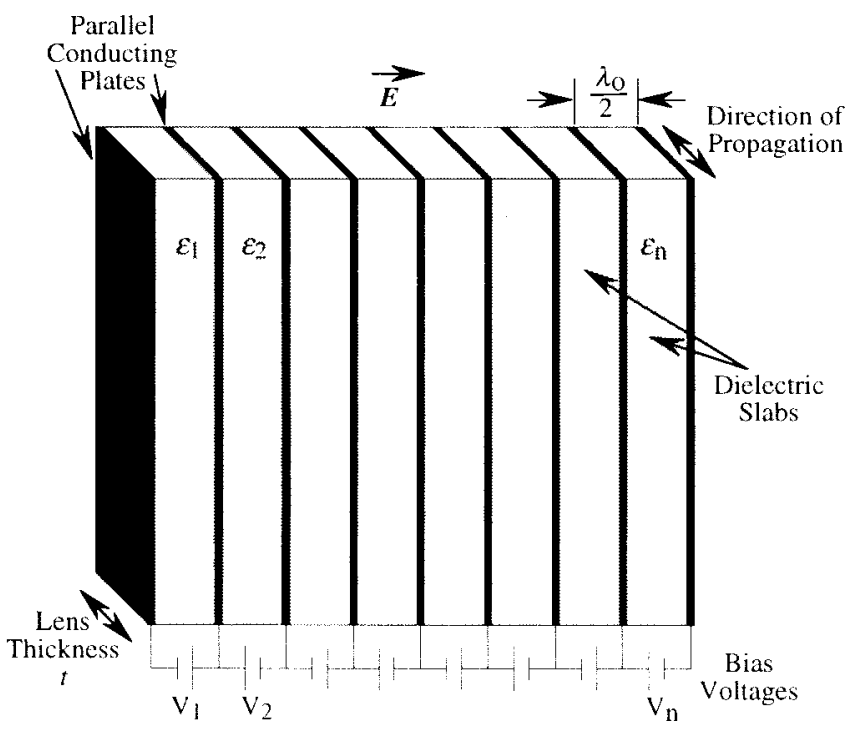

Fig. 1. Basic configuration of the ferroelectric lens.

has the further advantages of smaller lens thickness, higher power handling capability, simpler beam steering controls, and it uses less power to control the phase shift compared to the radant lens. Thus, it leads to low-cost phased arrays. However, it should be noted that the use of row-column steering may limit the level of sidelobes that can be achieved. For the present study, it is assumed that ultralow side lobes are not a requirement and that row-column phase control can be used to significantly reduce the phased-array cost.

\section{DESCRIPTION OF FERROELECTRIC LENS AND ITS OPERATION}

The main feature of the antennas that use ferroelectric material is the change of permittivity with an applied dc control voltage. A lens type antenna is discussed in this paper. Fig. 1 shows a dielectric lens made up of dielectric slabs sandwiched between conducting plates. Dielectric slabs are made up of ferroelectric material whose dielectric constant can be changed by applying and varying the dc electric field (dc voltage sources $V_{1}, V_{2} \ldots V_{n}$ are used for this purpose, as shown in Fig. 1). If a plane wave is incident on one side of the lens with RF electric field $\mathbf{E}$ normal to the conducting plates, the beam coming out on the other side of the lens can be scanned in the E-plane if a linear phase gradient is introduced along the $\mathbf{E}$-plane direction by adjusting the voltages $V_{1}, V_{2}, V_{3}, \cdots V_{n}$. The corresponding dielectric constants are shown as $\varepsilon_{1}, \varepsilon_{2}, \varepsilon_{3} \cdots \varepsilon_{n}$ in Fig. 1. 


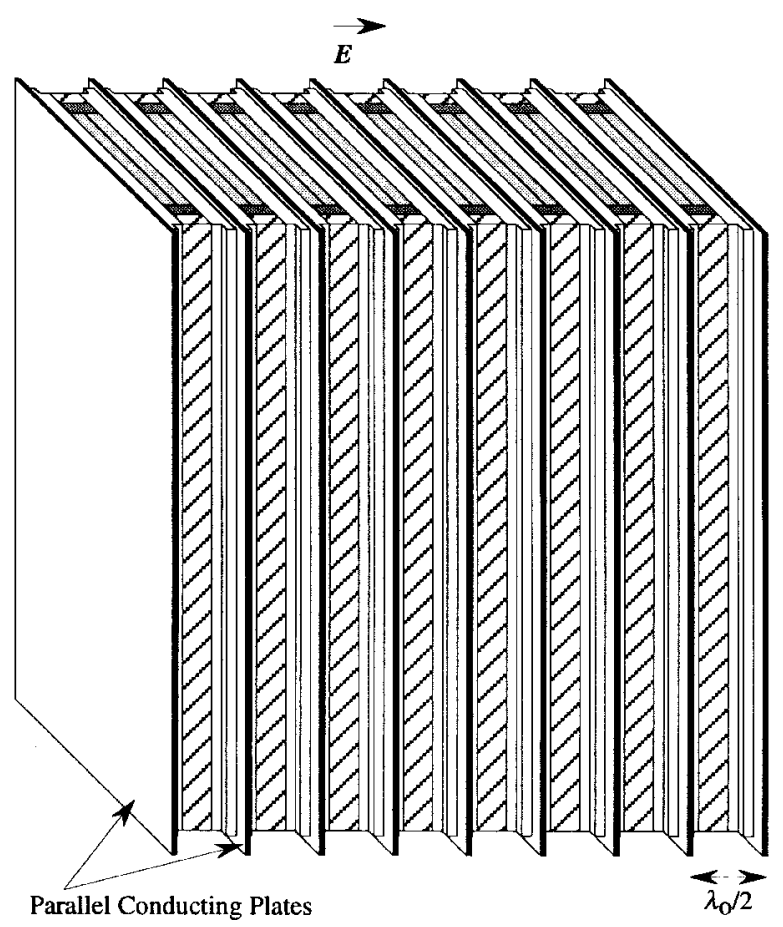

(a)

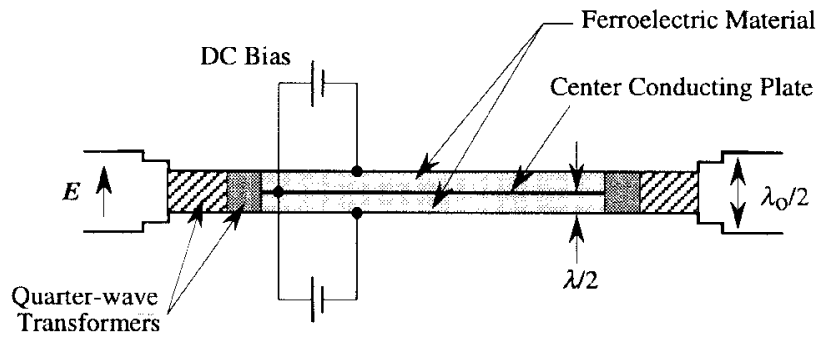

(b)

Fig. 2. (a) A more practical configuration of the ferroelectric lens. (b) Top view of one column.

Fig. 1 is used to illustrate the principle of operation of a ferroelectric lens. However, it has some limitations. To reduce the lens complexity and weight, the number of $\mathrm{dc}$ control voltages and the number of conducting plates need to be minimized. This can be achieved by selecting the separation between the conducting plates to be slightly less than $\lambda_{o} /\left(1+\sin \phi_{s}\right)$, similar to interelement spacing in any phased array in order to avoid grating lobes, where $\lambda_{o}$ is the free-space wavelength, and $\phi_{s}$ is the maximum scan angle. Normally, the space between the conducting plates would be $\sim \lambda_{\circ} / 2$. However, in a ferroelectric lens, this space is filled with a high dielectric constant ferroelectric material and so the space between the plates is much larger than $\lambda / 2$, where $\lambda$ is the wavelength in the ferroelectric. This means that higher order modes may propagate. Therefore, to eliminate the problem with higher order modes, the spacing between the conducting plates should be kept to less than $\lambda / 2$. In addition, there should be some type of impedance matching arrangement to match the lens surface to free-space.

Fig. 2 shows a more practical lens configuration. This configuration has several advantages over the basic configuration of Fig. 1. A stepped configuration is used for impedance matching to reduce the amount of dielectric material, to eliminate higher order mode propagation, and to reduce the bias voltage necessary to create a certain amount of dc electric field intensity within the ferroelectric material. Quarter-wave dielectric transformers are used for impedance matching of the empty waveguide region to the ferroelectric-loaded waveguide region. More than one transformer may be needed depending on the value of the dielectric constant of the ferroelectric material and the degree of matching needed. A further refinement is also shown in Fig. 2. This refers to the way the dc bias voltage is applied to the ferroelectric material. An additional center conducting plate is used to bifurcate the ferroelectric material. The dc voltage is applied between this center conducting plate (recessed with respect to parallel plates) and the parallel plates. Since the dielectric material is bifurcated, only half the dc voltage needs to be applied to produce the same dc electric field intensity. It is this dc electric field intensity that controls the dielectric constant of the ferroelectric. In addition, the parallel plates are at ground potential. This makes the handling of the lens safer.

The lens thickness needed to use the lens as a scanning antenna can be determined as follows. Let $t$ be the lens thickness as shown in Fig. 1. Let the dielectric constant of any one dielectric slab be $\varepsilon_{r, \max }$ when no bias voltage is applied. The dielectric constant of a ferroelectric decreases as the bias voltage is increased. Let $\varepsilon_{r, \min }$ be the dielectric constant when maximum dc bias is applied. The phase introduced by the slab when no bias voltage is applied is given by

$$
\phi_{\max }=\frac{2 \pi}{\lambda_{o}} t \sqrt{\varepsilon_{r, \max }} .
$$

Similarly, the phase introduced by the same slab when maximum bias voltage is applied is given by (note: dielectric constant decreases with applied bias voltage)

$$
\phi_{\min }=\frac{2 \pi}{\lambda_{o}} t \sqrt{\varepsilon_{r, \min }} .
$$

Then, the maximum phase change when the maximum bias voltage is applied is

$$
\phi_{\max }-\phi_{\min }=\frac{2 \pi}{\lambda_{o}} t\left(\sqrt{\varepsilon_{r, \max }}-\sqrt{\varepsilon_{r, \min }}\right) .
$$

For scanning applications, this maximum phase change should be equal to $2 \pi$ (as in the case of a phase shifter used in a scanning array), hence (3) becomes

$$
2 \pi=\frac{2 \pi}{\lambda_{o}} t\left(\sqrt{\varepsilon_{r, \max }}-\sqrt{\varepsilon_{r, \min }}\right) .
$$

So, the thickness of the ferroelectric material needed (in the direction of propagation) to obtain $360^{\circ}$ phase shift is

$$
t=\frac{\lambda_{o}}{\sqrt{\varepsilon_{r, \text { max }}}-\sqrt{\varepsilon_{r, \text { min }}}} .
$$

Tunability can be defined for a ferroelectric as the fractional change in the dielectric constant with applied dc bias voltage or

$$
\text { Tunability }=\frac{\varepsilon_{r, \max }-\varepsilon_{r, \min }}{\varepsilon_{r, \max }} .
$$




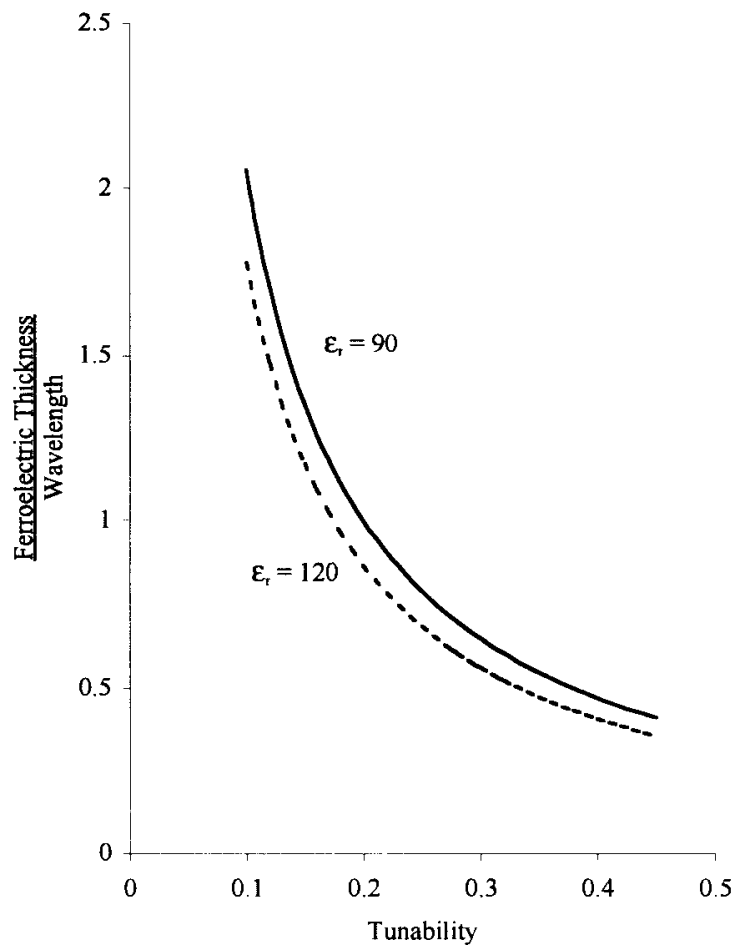

Fig. 3. Amount of ferroelectric needed for $360^{\circ}$ phase shift.

Now (5) can be written in terms of tunability as

$$
\frac{t}{\lambda_{o}}=\frac{1}{\sqrt{\varepsilon_{r, \max }}[1-\sqrt{1-\text { tunability }}} \text {. }
$$

So, the thickness of the ferroelectric material needed is a function of the dielectric constant and the tunability of the ferroelectric and the wavelength. The relationship shown in (7) is illustrated in Fig. 3 for typical $\varepsilon_{r}$ of 90 and 120. Additional discussion on this relationship is included later.

Also, it can be shown [5] that the attenuation constant for a uniform plane wave propagating in a low-loss dielectric is

$$
\alpha(\mathrm{dB})=27.3 \tan \delta \sqrt{\varepsilon_{r}} \frac{t}{\lambda_{\circ}}
$$

where $\tan \delta$ is the loss tangent. Using (7), we see that in order to obtain $360^{\circ}$ phase shift, the dielectric loss through the ferroelectric is

$$
\alpha(\mathrm{dB}) / 360^{\circ}=\frac{27.3 \tan \delta}{1-\sqrt{1-\text { tunability }}} .
$$

It may be noted that the lens loss is independent of the ferroelectric permittivity and depends only on its loss tangent and tunability. This equation is plotted in Fig. 4. It can also be approximated by using the Binomial series as

$$
\alpha(\mathrm{dB}) / 360^{\circ} \cong \frac{55 \tan \delta}{\text { tunability }} \text {. }
$$

In general, the ferroelectrics with higher $\varepsilon_{r}$ offer higher tunability, which is desired to reduce the lens thickness. However, the lens matching to free-space is easier for smaller $\varepsilon_{r}$. Therefore, a compromise is needed between reducing the lens thickness (to reduce overall lens size) and achieving reasonable impedance match to reduce reflections from the

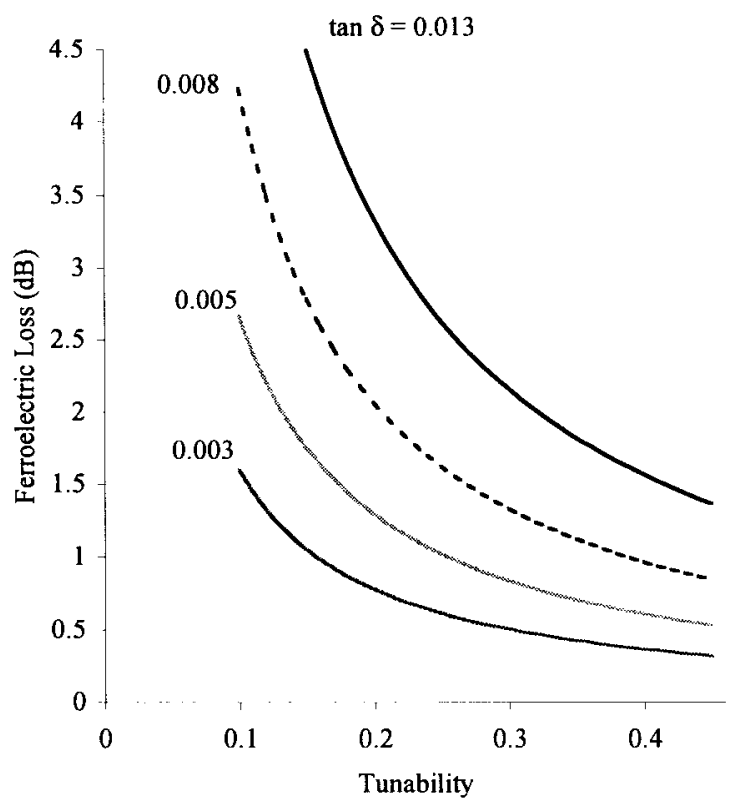

Fig. 4. Ferroelectric loss for $360^{\circ}$ phase shift.

lens surface. For a typical value of $\varepsilon_{r} \sim 100$, it is possible to obtain a tunability of $20 \%$ which results in a reasonable lens thickness of $\sim \lambda_{o}$ (e.g., $3 \mathrm{~cm}$ at $10 \mathrm{GHz}$ ), as shown in Fig. 3. For this reason, ferroelectric materials with $\varepsilon_{r} \sim 100$ were chosen for the experimental work which will be discussed later. In Fig. 4, it can be seen that $\tan \delta$ needs to be less than 0.005 to limit the lens loss to less than $1 \mathrm{~dB}$. The existing ferroelectric materials are a bit more lossy $(\tan \delta=0.008$ at $10 \mathrm{GHz}$ ) and further research is needed to reduce losses. Additional discussion will be included when the experimental results are discussed in a later part of the paper.

The ferroelectric lens offers electronic scanning in one plane. The lens proposed here can be fed by a nonscanning planar array, like a slotted waveguide array. A combination of slotted waveguide array with phase shifters and the lens proposed here can be used as a phased array which can scan in two planes. A space feed can be used with the combination of two lenses proposed here (with a polarization rotator in between) to scan the beam in two planes. The details of these alternatives are discussed in the next section.

\section{Phased-Array Configurations Using FERROELECTRIC LENS FOR 2-D SCANNING}

A single ferroelectric lens provides one-dimensional (1-D) electronic scanning. Two-dimensional (2-D) scanning can be achieved by cascading two ferroelectric lenses or using one ferroelectric lens in a hybrid configuration with a phased array which can scan in one plane.

Fig. 5 shows the cascading of two spatially orthogonal ferroelectric lenses. The first lens provides an elevation scan of a vertically polarized wave. A $90^{\circ}$ passive polarization rotator then rotates the electric field to become horizontally polarized. The second lens then provides the azimuth scanning of the horizontally polarized wave. In Fig. 5, a planar array is shown as the illuminator (or feed) for the dual lenses. A space 


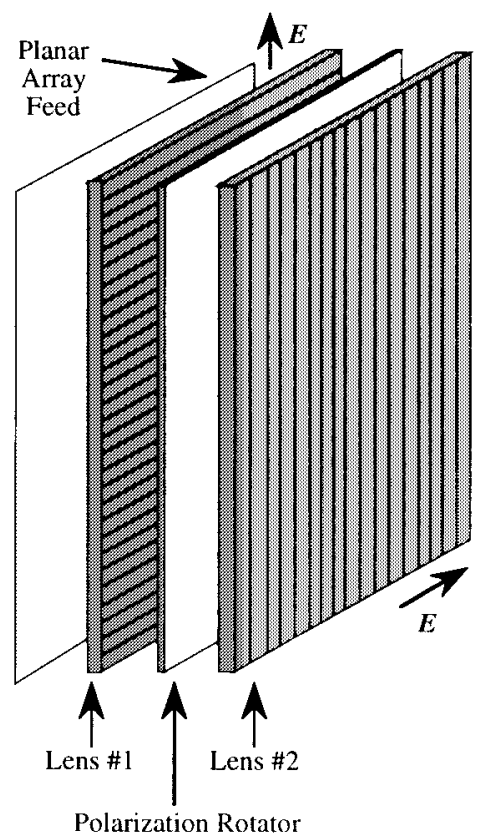

Fig. 5. Dual lens configuration with a planar array feed.

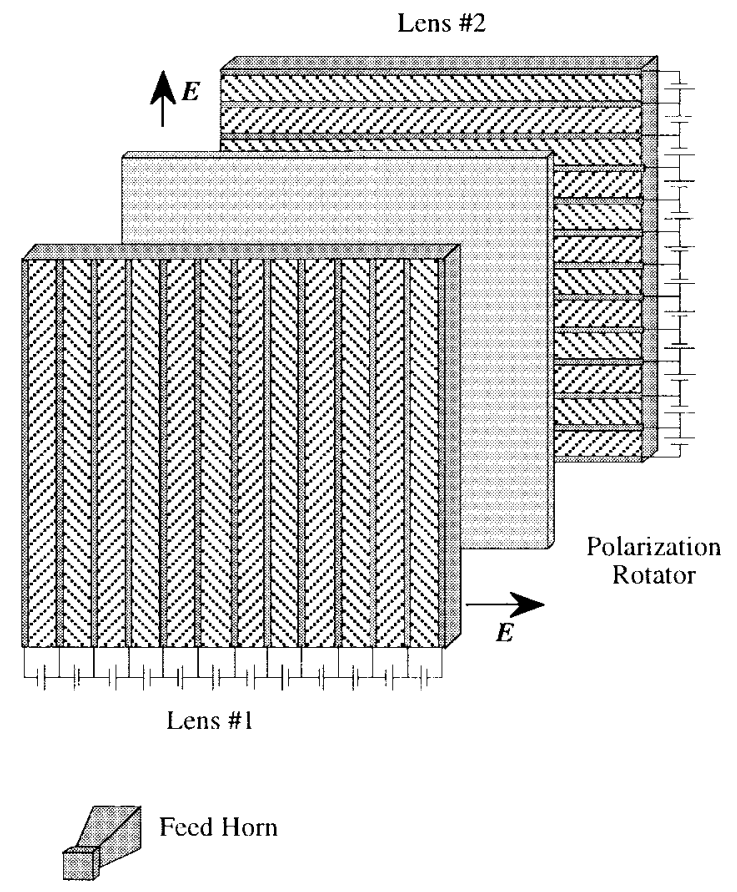

Fig. 6. Dual lens configuration with a space feed.

feed can also be used, as shown in Fig. 6, with the dual lens configuration. In this configuration, in addition to scanning the beam, row-column phase controls can also be used to correct the spherical phase errors due to the point space feed; however, this phase correction is not exact, but it is satisfactory for many applications. Spherical phase error corrections for an offset feed configuration is not possible with row-column phase controls. So, offset feeding is not recommended for dual lens configuration.

Another method of achieving 2-D scanning uses a hybrid technique in which a planar array with phase shifters provides

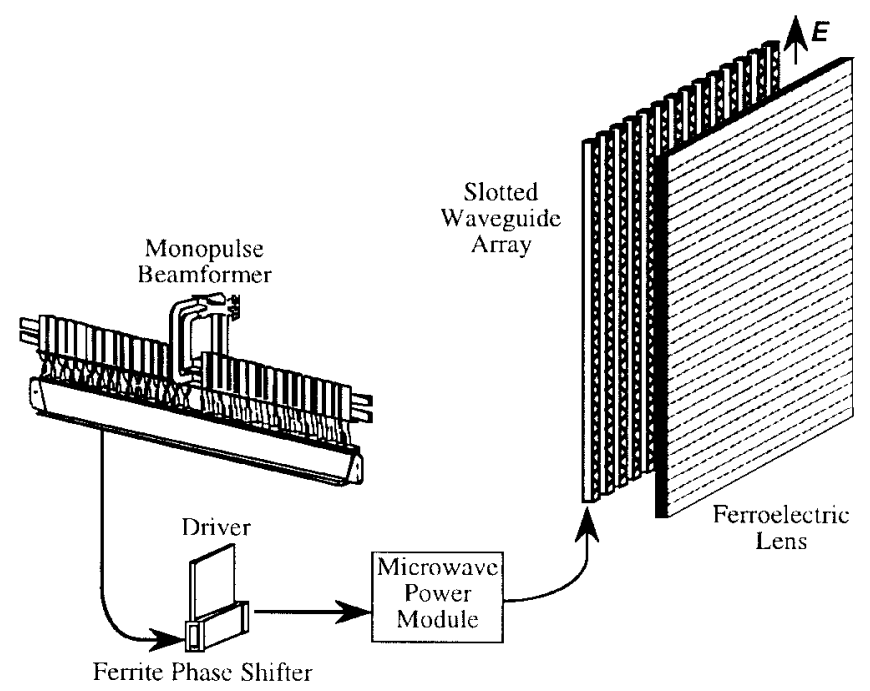

Fig. 7. Hybrid phased-array configuration for 2-D scanning.

scanning in one plane and the ferroelectric lens provides the scanning in the other plane. For example, consider a stacked slotted waveguide array with the slots in the narrow wall of the waveguide. Each waveguide is fed through a phase shifter at its input. Scanning in one plane is achieved with these phase shifters. Scanning in the orthogonal plane is obtained by placing a ferroelectric lens in front of the waveguide array, as shown in Fig. 7. For the reasons mentioned before, the cost of the phased arrays which use ferroelectric lens will be much less than the phased arrays that use phase shifter or $T / R$ module behind each radiating element.

\section{Ferroelectric Materials}

A ferroelectric material possesses spontaneous polarization. However, in our application, we use the material in the nonferroelectric (paraelectric) state above the Curie temperature. The high $\varepsilon_{r}$ and $\tan \delta$ of ferroelectric materials have limited their applications in phased arrays. Recent improvements in ferroelectric composite materials at the Army Research Laboratory (ARL), Aberdeen Proving Ground, MD, prompted renewed interest in developing phase shifters at microwave frequencies. The materials are bulk oxide-ceramic composites of barium strontium titanate oxide (BSTO). ARL has also developed a simple, low-cost processing technique for their manufacturing [6]-[8]. It involves mixing barium titanate $\left(\mathrm{BaTiO}_{3}\right)$ and strontium titanate $\left(\mathrm{SrTiO}_{3}\right)$ powders. The resulting BSTO powder is calcined and mixed with an oxide. After pressing and sintering, the composite is ready to be machined. Table I lists the properties of several ARL ferroelectric composites at room temperature. The first column indicates the $\mathrm{Ba} / \mathrm{Sr}$ ratio of the composition. The second column shows the weight percent oxide in the composition. The third column is the Curie temperature. The fourth column is the ferroelectric tunability at $2 \mathrm{~V} / \mu \mathrm{m}$. The lower the Curie temperature, the more paraelectric (and so the less tunable) the material becomes at room temperature. The last two columns list $\varepsilon_{r}$ and $\tan \delta$ measured at $1 \mathrm{kHz}$ and $10 \mathrm{GHz}$. Low frequency results were calculated from capacitance measurements. Measurements at 

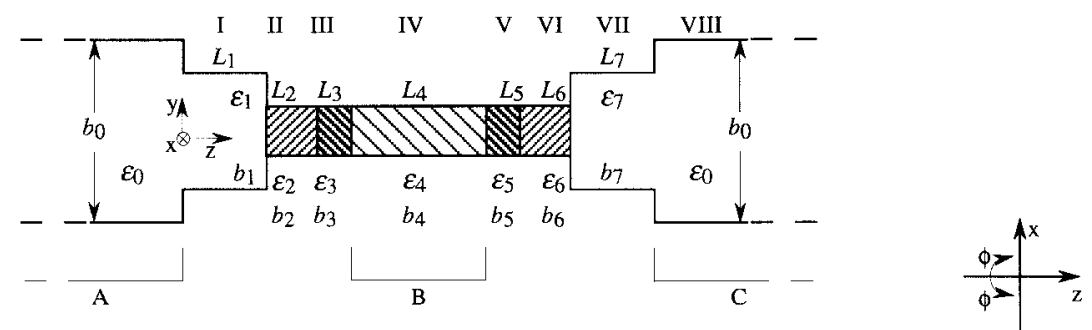

(a)

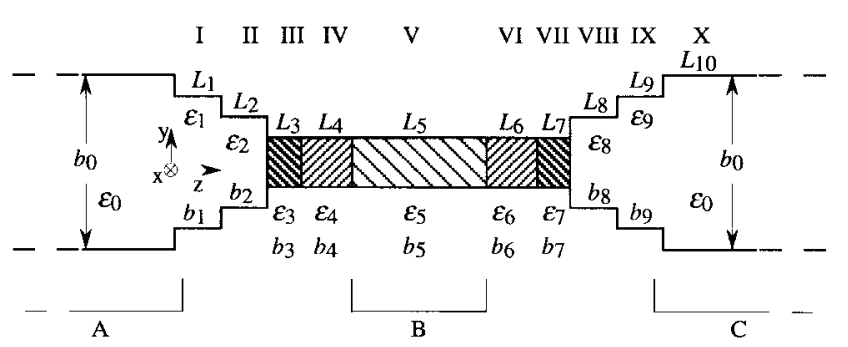

$L_{i}$ are lengths of waveguide sections

$b_{i}$ are the waveguide heights

$\varepsilon_{i}$ are the dielectric constants

(b)

Fig. 8. Matching networks. (a) Three-section matching network. (b) Four-section matching network.

TABLE I

Properties of ARL Bulk Ferroelectrics

\begin{tabular}{c|c|c|c|c|c}
\hline Material & $\begin{array}{c}\text { Oxide } \\
(w \mathrm{wt} \%)\end{array}$ & $\begin{array}{c}\text { Curie } \\
\mathrm{Temp}\end{array}$ & $\begin{array}{c}\text { Tunability } \\
(\%)\end{array}$ & $\begin{array}{c}\varepsilon_{\mathrm{r}} \\
1 \mathrm{kHz} / 10 \mathrm{GHz}\end{array}$ & $\begin{array}{c}\tan \delta \times 10^{-3} \\
1 \mathrm{kHz} / 10 \mathrm{GHz}\end{array}$ \\
\hline $\mathrm{Ba}_{0.50} \mathrm{Sr}_{0.50} \mathrm{TiO}_{3}$ & 0 & $-25^{\circ} \mathrm{C}$ & 25.5 & $1908 / 1099$ & $20.0 / 18.9$ \\
\hline $\mathrm{Ba}_{0.50} \mathrm{Sr}_{0.50} \mathrm{TiO}_{3}$ & 20 & $-55^{\circ} \mathrm{C}$ & 6.44 & $592 / 616$ & $0.73 / 8.7$ \\
\hline $\mathrm{Ba}_{0.50} \mathrm{Sr}_{0.50} \mathrm{TiO}_{3}$ & 30 & $-70^{\circ} \mathrm{C}$ & 5.82 & $414 / 463$ & $0.7 / 8.4$ \\
\hline $\mathrm{Ba}_{0.50} \mathrm{Sr}_{0.50} \mathrm{TiO}_{3}$ & 60 & $-95^{\circ} \mathrm{C}$ & 3.66 & $78 / 84$ & $0.76 / 6.5$ \\
\hline $\mathrm{Ba}_{0.55} \mathrm{Sr}_{0.45} \mathrm{TiO}_{3}$ & 30 & $-45^{\circ} \mathrm{C}$ & 9.54 & $478 / 527$ & $0.75 / 12.1$ \\
\hline $\mathrm{Ba}_{0.55} \mathrm{Sr}_{0.45} \mathrm{TiO}_{3}$ & 60 & $-50^{\circ} \mathrm{C}$ & 6.46 & $95 / 100$ & $0.34 / 7.9$ \\
\hline $\mathrm{Ba}_{0.60} \mathrm{Sr}_{0.40} \mathrm{TiO}_{3}$ & 60 & $-55^{\circ} \mathrm{C}$ & 9.99 & $117 / 118$ & $1.48 / 12.9$ \\
\hline
\end{tabular}

$10 \mathrm{GHz}$ were performed by Dr. R. Geyer at the National Institute of Standards and Technology and confirmed with our measurements. We see that, in general, tunability, $\varepsilon_{r}$, and $\tan \delta$ decrease with decreasing $\mathrm{Ba}$ content; they also decrease with increasing oxide content. After oxide is added, $\varepsilon_{r}$ changes much less with frequency. As expected for ceramics, $\tan \delta$ increases with frequency; however, it is far lower than that of any other tunable ferroelectrics. Measurements at higher bias voltages indicate that tunability increases linearly with an increase in bias voltage.

\section{TheORETICAL ANALYSIS AND ExPERIMENTAL REsults}

For the theoretical analysis of the ferroelectric lens, an individual section between two conducting parallel plates of the lens can be considered as one column of a phased array. Initially, the column height is considered infinite in extent and, hence, it becomes a 2-D parallel-plate waveguide with electric field normal to the plates as shown in Fig. 2(b). The lens column fed by a parallel-plate waveguide was evaluated assuming transverse electromagnetic (TEM) mode propagation. To eliminate higher order mode propa- gation, the ferroelectric slab thickness between one of the parallel plates and the central conducting plate is limited to less than $\lambda / 2$, as discussed earlier. Theoretical analysis was also performed using Touchstone ${ }^{\circledR}$, a commonly available software, for a rectangular waveguide with a cross section shown in Fig. 2(b). The analysis was done assuming different column heights (waveguide widths). For widths larger than $4 \lambda_{o}$, the rectangular waveguide results matched well with parallel plate results. For practical reasons, most of the initial measurements were performed in a reduced height $X$-band rectangular (WR90) waveguide. Theoretical and experimental results for the waveguide configuration along with a brief description of the ferroelectric lens and its principle of operation have been reported earlier [8]-[11]. This paper includes the detailed theoretical analysis of the ferroelectric lens along with the extensive experimental results. Theoretical analysis also indicated that the results are better for a parallel-plate waveguide than with a rectangular waveguide.

In this paper, for theoretical analysis and numerical results, the geometry was restricted to be 2-D since each column of the ferroelectric lens is predominantly 2-D as discussed earlier. Because the lens is to be fed with a linearly polarized field with the electric field normal to the conducting plates, the lens was analyzed only for that case. A single column of the lens fed by a parallel-plate waveguide was evaluated as a parallelplate waveguide. Therefore, the results presented here are for a single column of the lens and do not include the effects of mutual coupling between columns.

\section{A. Analytical Solution}

We have considered two impedance matching networks to match the lens surface to free-space over a broad range of frequencies. Both networks use multisection impedance matching transformers. They are shown in Fig. 8, which are the top views of one column. The matching network in Fig. 8(a) uses three transformers. The matching network in 
Fig. 8(b) uses four transformers. Each transformer is a section of a parallel-plate waveguide. In Fig. 8(a)

$$
\begin{aligned}
& L_{i}=L_{8-i}, \quad b_{i}=b_{8-i}, \quad \varepsilon_{i}=\varepsilon_{8-i}, \quad \varepsilon_{4}=\varepsilon_{r}, \\
& b_{2}=b_{3}=b_{4}=b
\end{aligned}
$$

where $i=1,2,3$.

In Fig. 8(b)

$$
\begin{aligned}
& L_{i}=L_{10-i}, \quad b_{i}=b_{10-i}, \quad \varepsilon_{i}=\varepsilon_{10-i}, \quad \varepsilon_{5}=\varepsilon_{r}, \\
& b_{3}=b_{4}=b_{5}=b
\end{aligned}
$$

where $i=1,2,3,4$.

$L_{i}$ are the lengths of the waveguide sections, $b_{i}$ are the heights (separations between the parallel plates) of the waveguide sections and $\varepsilon_{i}$ are the dielectric constants of the dielectrics completely filling the waveguide sections. $\varepsilon_{r}$ is the dielectric constant of the ferroelectric material. Since the input and output sections of the lens are identical (empty parallelplate waveguide), the same matching network can be used at both ends. The matching network provides impedance matching between the empty semi-infinite parallel-plate waveguide ( $A$ and $C$ in Fig. 8) and the waveguide section completely filled with the ferroelectric ( $B$ in Fig. 8). The heights of waveguides $A, B$, and $C$ are chosen to assure that only the dominant TEM mode will propagate in the waveguides and that grating lobes are not formed when an antenna is designed by arraying several columns together. Because waveguide $B$ is filled with a high dielectric constant material, its height is chosen to be much smaller than the height of waveguides $A$ and $C$ to eliminate higher order mode problems. The heights of waveguides $A$ and $C$ are the same and are approximately $\lambda_{\circ} / 2$ at the highest frequency of operation in order to avoid grating lobes.

The matching network of Fig. 8(a) uses a design with three transformers where the first transformer is an empty parallelplate waveguide whose height is between that of waveguide $A$ (or $C$ ) and $B$. The other two transformers are parallel-plate waveguides completely filled with dielectric materials whose relative permittivities $\left(\varepsilon_{r}\right)$ are between that of air (or unity) and that of the ferroelectric. Dielectric constant of the first transformer is less than that of the second transformer. The heights of these two transformers are the same as the height of waveguide $B$. The matching network of Fig. 8(b) uses four transformers where the first two transformers are empty parallel-plate waveguides whose heights are between that of waveguide $A$ (or $C$ ) and $B$. The height of the first transformer is larger than the height of the second transformer. The other two transformers are parallel-plate waveguides completely filled with dielectric materials, whose relative permittivities are between that of air (or unity) and that of the ferroelectric. The heights of these two transformers are the same as the height of waveguide $B$. Dielectric constant of the first transformer is less than that of the second transformer.

Since a single ferroelectric lens provides electronic scanning in one plane, another lens or antenna is needed to scan in the second plane. If the first antenna (the one closer to the transmitter/receiver) is a lens, the electromagnetic wave is incident normally on this lens from the feed. This lens will create a phase gradient in one plane and its radiation will be incident obliquely onto the second lens. For the theoretical analysis of one column, we assume that the dominant TEM mode is obliquely incident in the $H$-plane. The electric field is orthogonal to the waveguide plates ( $y$-direction). The magnetic field is in the plane parallel to the waveguide walls. The oblique incidence angle is measured with respect to the $z$ axis. This is the scan angle of the first antenna. A time dependency of $\exp (j \omega t)$ is assumed, and the waveguide walls are assumed to be perfect conductors.

As a plane TEM wave is obliquely incident on the matching network from waveguide $A$, some of the energy associated with the incident wave will be reflected back to waveguide $A$. We will derive an analytical solution for the reflection coefficient and use this solution to design the optimum matching network which minimizes the magnitude of the reflected field for a range of incidence angles over the frequency band of interest and for the required change of the ferroelectric dielectric constant to obtain $360^{\circ}$ differential phase shift. The case of normal incidence represents a special case of oblique incidence where the incidence angle is zero.

The optimization algorithms were devised and computer programs have been written based on the analytical solutions. As optimization parameters, computer programs use lengths of the transformers, height(s) of the empty waveguide transformer(s), and the dielectric constants of the materials in the last two transformers.

First, consider the matching network which uses four transformers shown in Fig. 8(b). Assuming that the dominant TEM mode is obliquely incident, the electric field in waveguide $A$ can be written as

$$
E_{y}^{(i)}=A_{o} \exp \left[-j k_{o}(z \cos (\phi)+x \sin (\phi))\right]
$$

where $A_{o}$ is the amplitude of the incident wave, $k_{o}$ is the free-space wave number, and $\phi$ is the oblique incidence angle. The magnetic field components of the incident TEM mode are defined using Maxwell's equations as

$$
H_{x}=\frac{1}{j k_{o} \mu_{o}} \frac{\partial E_{y}}{\partial z}
$$

and

$$
H_{z}=-\frac{1}{j k_{o} \mu_{o}} \frac{\partial E_{y}}{\partial x}
$$

where $\mu_{o}$ is the free-space permeability. Based on (11a) and (12a), the $x$ component of the incident wave's magnetic field can be expressed as

$$
H_{x}^{(i)}=-A_{o} \frac{\cos (\phi)}{\mu_{o}} \exp \left[-j k_{o}(z \cos (\phi)+x \sin (\phi))\right] .
$$

Reflection occurs at the interface between waveguide $A$ and the matching network. The angle of reflection is equal to the angle of incidence. The reflected wave's field components in waveguide $A$ are

$$
E_{y}^{(r)}=R_{o} \exp \left[j k_{o}(z \cos (\phi)-x \sin (\phi))\right]
$$

and

$$
H_{x}^{(r)}=R_{o} \frac{\cos (\phi)}{\mu_{o}} \exp \left[j k_{o}(z \cos (\phi)-x \sin (\phi))\right]
$$

where $R_{o}$ is the amplitude of the reflected wave. 
Since the input and output matching networks are identical, the angle at which the plane wave propagates in waveguide $C$ [area $\mathrm{X}$ in Fig. 8(b)] is equal to the angle at which the plane wave was incident in the waveguide $A$. The electric and magnetic fields of the dominant mode in area $\mathrm{X}$ can be written as

$$
E_{y}^{(10)}=T_{10} \exp \left[-j k_{o}(z \cos (\phi)+x \sin (\phi))\right]
$$

and

$$
H_{x}^{(10)}=-T_{10} \frac{\cos (\phi)}{\mu_{o}} \exp \left[-j k_{o}(z \cos (\phi)+x \sin (\phi))\right]
$$

where $T_{10}$ is the amplitude of the wave in waveguide $C$ (area $\mathrm{X})$.

In area IX of Fig. 8(b), the electromagnetic fields can be represented as a sum of two waves: the first wave travels in the positive $z$ direction with an amplitude $T_{9}$; the second wave travels in the negative $z$ direction with an amplitude $R_{9}$. The fields can be written as

$$
\begin{aligned}
E_{y}^{(9)}= & T_{9} \exp \left[-j k_{o}(z \cos (\phi)+x \sin (\phi))\right] \\
& +R_{9} \exp \left[-j k_{o}(-z \cos (\phi)+x \sin (\phi))\right]
\end{aligned}
$$

and

$$
\begin{aligned}
H_{x}^{(9)}= & \frac{\cos (\phi)}{\mu_{o}}\left\{-T_{9} \exp \left[-j k_{o}(z \cos (\phi)+x \sin (\phi))\right]\right. \\
& \left.+R_{9} \exp \left[-j k_{o}(-z \cos (\phi)+x \sin (\phi))\right]\right\}
\end{aligned}
$$

$T_{9}$ and $R_{9}$ are to be determined from the boundary conditions at the interfaces between areas VIII and IX and areas IX and $\mathrm{X}$. The oblique angles of the waves propagating in area IX are the same as in area $\mathrm{X}$ because both areas are filled with the same dielectric (air).

The boundary conditions require that the tangential components of electric and magnetic field be continuous at all discontinuity interfaces. The interface between areas IX and $\mathrm{X}$ is at

$$
z=d_{9} \equiv \sum_{i=1}^{9} L_{i}
$$

and the boundary conditions are

$$
E_{y}^{(10)}= \begin{cases}0 & b_{9} / 2 \leq y \leq b_{\circ} / 2 \\ E_{y}^{(9)} & -b_{9} / 2 \leq y \leq b_{9} / 2 \\ 0 & -b_{\circ} / 2 \leq y \leq-b_{9} / 2\end{cases}
$$

and

$$
H_{x}^{(10)}=H_{x}^{(9)} \quad-b_{9} / 2 \leq y \leq b_{9} / 2 .
$$

Substituting electric field expressions from (14a) and (15a) into the boundary condition (17a), followed by integration over the interval $\left[-b_{o} / 2 \leq y \leq b_{o} / 2\right]$ leads to

$$
\begin{aligned}
& T_{10} b_{o} \exp \left[-j k_{o} d_{9} \cos (\phi)\right] \\
& \quad=b_{9}\left\{T_{9} \exp \left[-j k_{o} d_{9} \cos (\phi)\right]+R_{9} \exp \left[j k_{o} d_{9} \cos (\phi)\right]\right\} .
\end{aligned}
$$

Similarly, substituting magnetic field expressions from (14b) and (15b) into the boundary condition (17b) followed by integration over the interval $\left[-b_{9} / 2 \leq y \leq b_{9} / 2\right]$ leads to

$$
\begin{aligned}
& -T_{10} \exp \left[-j k_{o} d_{9} \cos (\phi)\right] \\
& \quad=-T_{9} \exp \left[-j k_{o} d_{9} \cos (\phi)\right]+R_{9} \exp \left[j k_{o} d_{9} \cos (\phi)\right] .
\end{aligned}
$$

Dividing (18a) by (18b), we get

$$
R_{9}=T_{9} Q_{9} \exp \left[-j k_{o} 2 d_{9} \cos (\phi)\right]
$$

where

$$
Q_{9}=\frac{\left[b_{\circ} / b_{9}-1\right]}{\left[b_{\circ} / b_{9}+1\right]}
$$

Using (19)-(20), the electromagnetic field components $E_{y}^{(9)}$ and $H_{x}^{(9)}$ can be expressed as

$$
\begin{aligned}
E_{y}^{(9)}= & T_{9} \exp \left[-j k_{o}\left(x \sin (\phi)+d_{9} \cos (\phi)\right)\right] \\
& \times\left\{\exp \left[-j k_{o}\left(z-d_{9}\right) \cos (\phi)\right]\right. \\
& \left.+Q_{9} \exp \left[j k_{o}\left(z-d_{9}\right) \cos (\phi)\right]\right\}
\end{aligned}
$$

and

$$
\begin{aligned}
H_{x}^{(9)}= & T_{9} \frac{\cos (\phi)}{\mu_{o}} \exp \left[-j k_{o}\left(x \sin (\phi)+d_{9} \cos (\phi)\right)\right] \\
& \times\left\{-\exp \left[-j k_{o}\left(z-d_{9}\right) \cos (\phi)\right]\right. \\
& \left.+Q_{9} \exp \left[j k_{o}\left(z-d_{9}\right) \cos (\phi)\right]\right\} .
\end{aligned}
$$

Continuing this process of writing the TEM field components in each consecutive area and applying boundary conditions at each interface, we arrive at the expression for the dominant mode-reflection coefficient in waveguide $A$ as reported earlier [12]

$$
R_{o}=\frac{f_{1}+b_{o} / b_{1}}{f_{1}-b_{o} / b_{1}}
$$

where

$$
\begin{aligned}
Q_{i} & =\frac{\left(v_{i} / v_{i+1}\right) f_{i+1}+\left(b_{i} / b_{i+1}\right)}{\left(v_{i} / v_{i+1}\right) f_{i+1}-\left(b_{i} / b_{i+1}\right)} \\
f_{i} & =\frac{1+Q_{i} \exp \left[-j k_{o} v_{i} 2 L_{i}\right]}{-1+Q_{i} \exp \left[-j k_{o} v_{i} 2 L_{i}\right]} \\
v_{i} & =\sqrt{\varepsilon_{r i}-\sin ^{2}(\phi)} \\
i & =1, \ldots, M-1 \\
f_{M} & =-1 \\
v_{M} & =\cos (\phi)
\end{aligned}
$$

where $M=10$. In order to determine the reflection coefficient for the matching network, which uses four transformers $f_{1}$ is needed. It is computed iteratively as follows. Initially, $f_{10}=-1$ is used to compute $Q_{9}$ and then $f_{9}$ is computed; $f_{9}$ is used to compute $Q_{8}$ and so on until $f_{1}$ can be computed.

A similar solution was derived for the matching network which uses three transformers shown in Fig. 8(a). It is expressed by the same set of equations as (22)-(28), except now with $M=8$. 


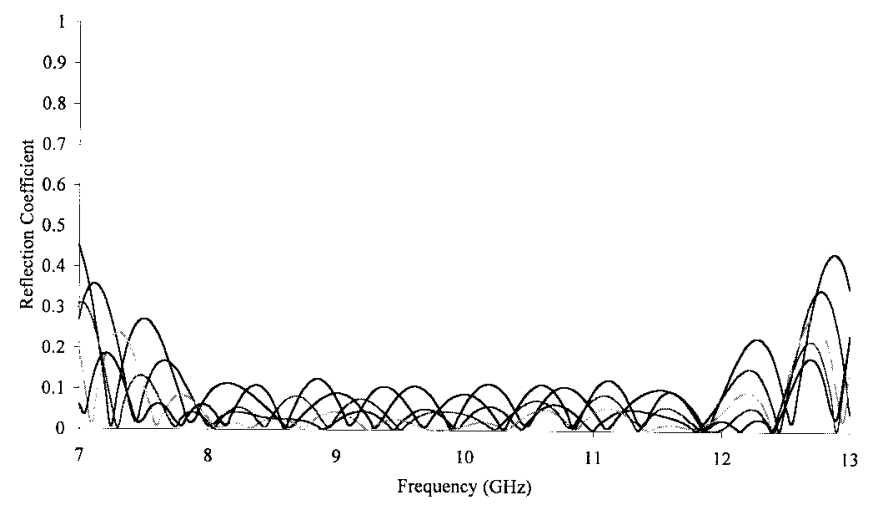

Fig. 9. Reflection coefficient for normal incidence with three-section matching network for various dielectric constants $\left(\varepsilon_{r}=80,90,100,110\right.$, and 120).

\section{B. Optimization and Numerical Results}

Using the analytical solutions that were developed in the previous section, optimization algorithms based on the minimax approach have been devised and computer programs have been written to implement these algorithms [12]. The programs optimize the length of each transformer. For the transformers that use empty parallel-plate sections, the height is also optimized. For the last two transformers that use dielectric materials, the programs evaluate various combinations of dielectric constants; we use only the dielectric constants of materials that can readily be obtained from manufacturers. The height of the waveguide containing the last two transformers is the same as the height of the waveguide containing the ferroelectric material.

The programs provide the optimum values of the transformer parameters that minimize the magnitude of the reflected field for a range of incidence angles over the frequency band of interest and for the required variation of the ferroelectric dielectric constant to obtain $360^{\circ}$ differential phase shift.

The matching network that uses three transformers [Fig. 8(a)] does not provide a low enough reflection coefficient for a wide range of incidence angles. However, for normal incidence, the reflection coefficient is sufficiently low. The optimization was conducted assuming that the dielectric constant of the ferroelectric varies from 120 to 80 over a frequency range of $8-12 \mathrm{GHz}\left(40 \%\right.$ bandwidth) and that $b_{0}=$ $0.4^{\prime \prime} ; b_{2}=b_{3}=b_{4}=0.1^{\prime \prime} ; \varepsilon_{1}=\varepsilon_{o}$. The computed results for normal incidence are shown in Fig. 9. The corresponding optimum parameters are: $L_{1}=0.2956^{\prime \prime} ; L_{2}=0.1860^{\prime \prime}$; $L_{3}=0.0505^{\prime \prime} ; b_{1}=0.2345^{\prime \prime} ; \varepsilon_{2}=2.54 ; \varepsilon_{3}=35$. These results were compared with the results obtained by using commercially available software "Touchstone ${ }^{\circledR}$ " for a rectangular waveguide. When the width of the rectangular waveguide is chosen to be greater than $4 \lambda_{o}$, the results obtained with Touchstone $\AA$ are identical to results obtained from our computer program for the parallel-plate waveguide structure under consideration.

The matching network that uses four transformers [Fig. 8(b)] provides sufficiently low reflection coefficient even for the case of oblique incidence. The optimization was performed for incidence angle range of $0^{\circ}-55^{\circ}$ and

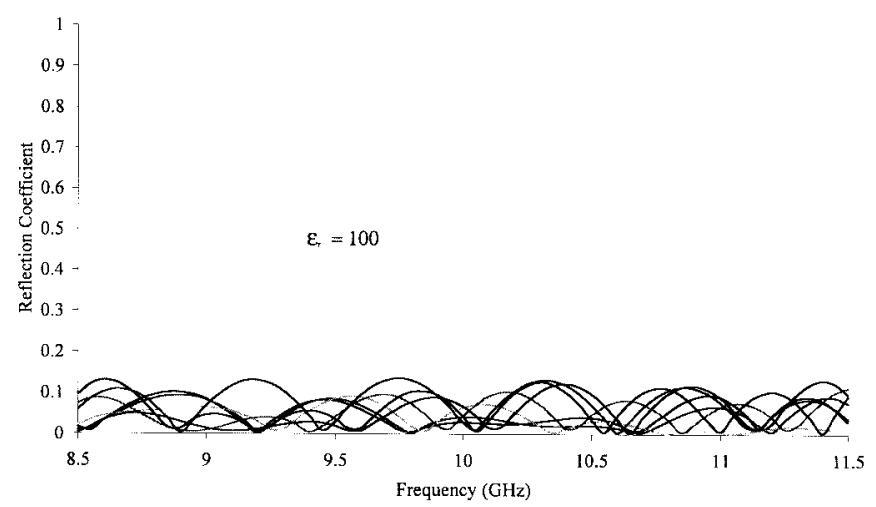

(a)

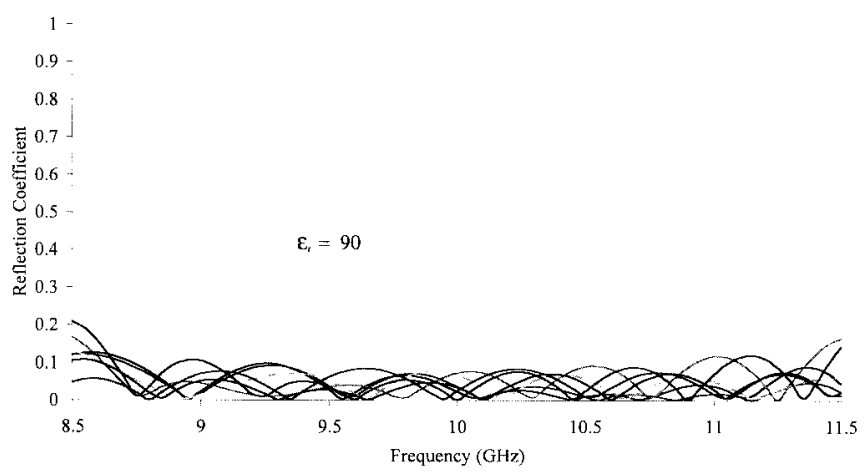

(b)

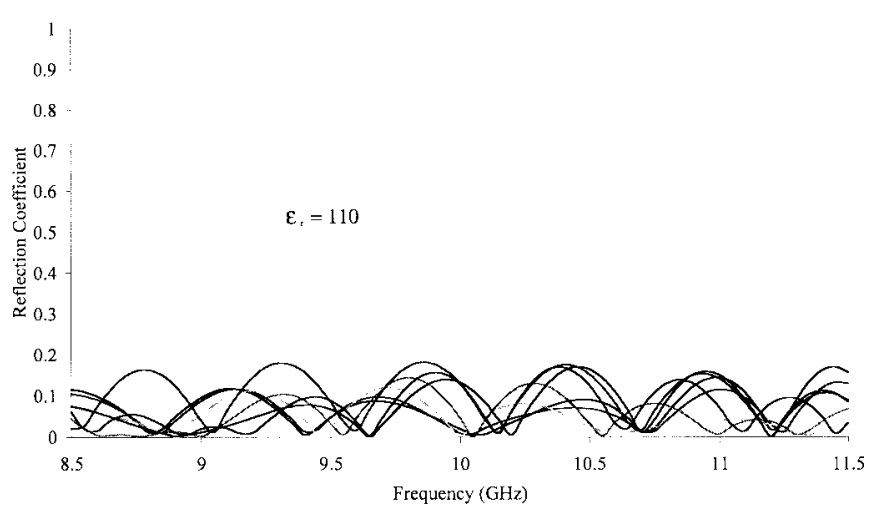

(c)

Fig. 10. (a) Reflection coefficient for $\varepsilon_{r}=100$, several incidence angles $\left(\phi=0,10,20,30,40,50\right.$, and $\left.55^{\circ}\right)$, and $30 \%$ bandwidth with four-section matching network. (b) Reflection coefficient for $\varepsilon_{r}=90$, several incidence angles $\left(\phi=0,10,20,30,40,50\right.$, and $\left.55^{\circ}\right)$, and $30 \%$ bandwidth with four-section matching network. (c) Reflection coefficient for $\varepsilon_{r}=110$, several incidence angles $\left(\phi=0,10,20,30,40,50\right.$, and $\left.55^{\circ}\right)$, and $30 \%$ bandwidth with four-section matching network.

for frequency range of $8.5-11.5 \mathrm{GHz}$ (30\% bandwidth). The dielectric constant of the ferroelectric was assumed to be 100. Variation of the dielectric constant was not used in the optimization although it can be included. Again, $b_{0}=0.4^{\prime \prime} ; b_{3}=b_{4}=b_{5}=0.1^{\prime \prime} ; \varepsilon_{1}=\varepsilon_{2}=\varepsilon_{0}$. The optimum parameters are: $L_{1}=0.3567^{\prime \prime} ; L_{2}=0.3866^{\prime \prime}$; $L_{3}=0.2063^{\prime \prime} ; L_{4}=0.0539^{\prime \prime} ; b_{1}=0.2978^{\prime \prime} ; b_{2}=0.1636^{\prime \prime}$; $\varepsilon_{3}=2.54 ; \varepsilon_{4}=30$. The reflection coefficient is calculated to be less than 0.13, as shown in Fig. 10(a); seven curves are plotted corresponding to seven incidence angles. With all other parameters kept constant, the dielectric constant 


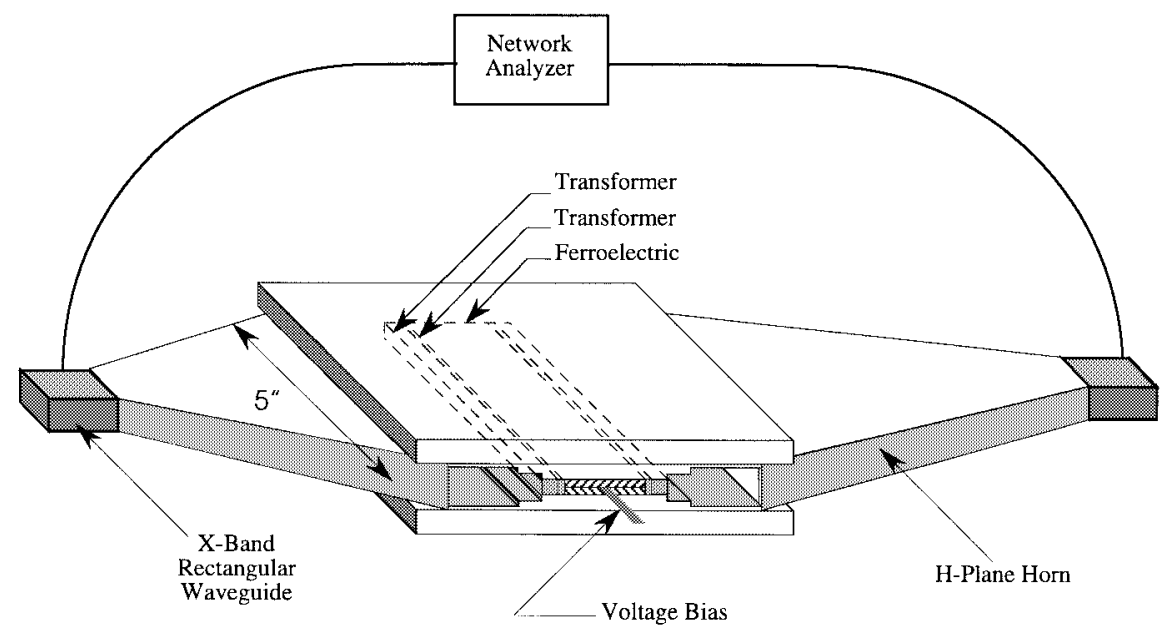

Fig. 11. Experimental setup for one column of the lens.

of the ferroelectric was changed to 90 and 110 to examine the effect of applying dc bias voltage on the reflection coefficient. The results are shown in Fig. 10(b) and (c); there is no significant change in the magnitude of the reflection coefficient compared to that of Fig. 10(a). These results indicated that the lens is well matched even after applying dc bias voltage to scan the beam.

Theoretical results presented in this section indicated that a ferroelectric lens can be designed, which is well matched over large scan angles and broad frequency range. The reflection coefficient can be reduced further (or the matching can be improved) by decreasing the frequency bandwidth and/or the range of incidence angles over which the lens operates. If necessary, the matching can also be improved by increasing the number of transformers in the matching network.

\section{Experimental Results}

The measurement setup is shown in Fig. 11. An HP 8720B Network Analyzer is connected to a WR90 ( $X$-band rectangular) waveguide through a coax-to-waveguide transition. The width of the WR90 is flared from 0.9 in to 5 in to create a $H$-plane horn, which feeds the parallel-plate column. The column uses the matching network with three transformers. The transformer parameters were given in the previous section and the reflection coefficient was shown in Fig. 9. The network analyzer measures the $s$ parameters of the column as a function of frequency and dc bias voltage. The ferroelectrics were 1 in long (in the direction of propagation), 0.05 in high and 5 in wide. Experiments were performed with $\mathrm{Ba}_{0.55} \mathrm{Sr}_{0.45} \mathrm{TiO}_{3}$ with $60 \%$ oxide. This material offered a good compromise among $\varepsilon_{r}$, $\tan \delta$, and tunability. Properties of this material are given in Table I; $\varepsilon_{r}=100$ and $\tan \delta=0.0079$ at 10 GHz. Fig. 12 shows the measured transmission and reflection coefficients of this material. Since the input and output reflection coefficients are almost identical, only one is shown. The reflection coefficient is sufficiently small over a wide frequency band as the theory had predicted in Fig. 9. Fig. 12 shows that the loss increases with frequency; this is due to two reasons. First, $\tan \delta$ increases with frequency, which is expected for ceramics; second, the electrical length (in

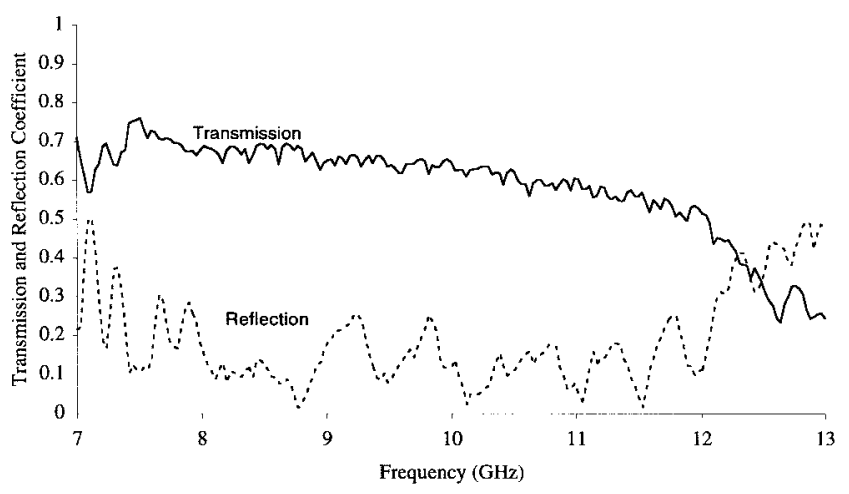

Fig. 12. Measured voltage transmission and reflection coefficients.

terms of wavelengths) of the ferroelectric in the direction of propagation increases with frequency since the physical length is kept constant (1").

Using Fig. 12, our calculations showed that the dielectric loss due to the ferroelectric material varied from approximately $2 \mathrm{~dB}$ at $9 \mathrm{GHz}$ to $2.5 \mathrm{~dB}$ at $11 \mathrm{GHz}$ for obtaining a phase shift of $360^{\circ}$ at $9 \mathrm{GHz}$. At higher frequencies, the phase shift is proportionately larger. This loss is more than what is desired in any practical application. We are, however, actively participating in a U.S. Department of Defense program to improve ferroelectric materials. The Frequency Agile Materials for Electronics (FAME) Program is being managed and directed by the Defense Advanced Research Projects Agency (DARPA). The goal of the FAME program is to provide an order of magnitude enhancement in component performance over current state of the art. We are participating in two DARPA contracts (one with Raytheon Company and the other with Northrop Grumman Corporation) to reduce the dielectric loss in the ferroelectric materials below $1 \mathrm{~dB}$ for $360^{\circ}$ phase shift.

For high-power applications, even 1-dB loss may create temperature increase in the ferroelectric material. However, the ferroelectric lens uses thin slabs of ferroelectric material with large empty space between the adjacent waveguide sections containing ferroelectric slabs. This configuration provides a natural duct for forced air cooling. Our analysis indicated that 


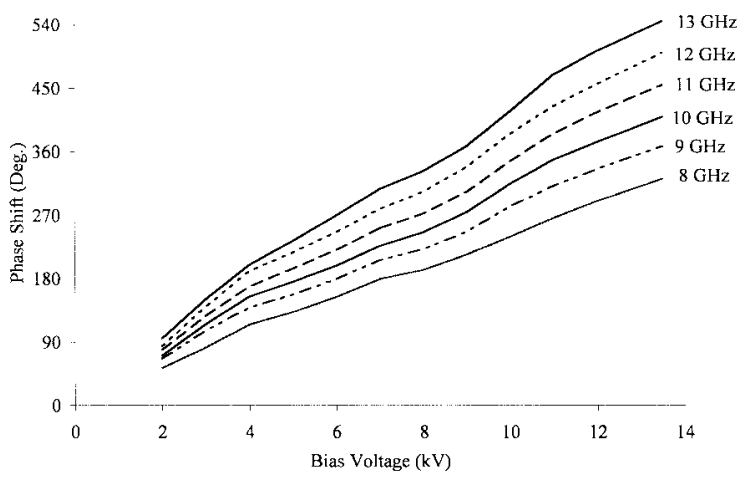

Fig. 13. Measured phase shift as a function of the bias voltage for various frequencies.

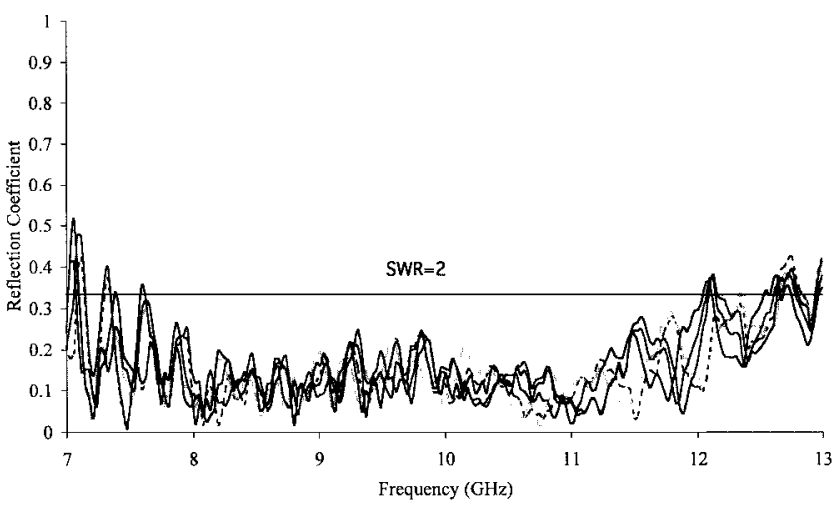

Fig. 14. Measured reflection coefficients as a function of frequency for various bias voltages $(0-13.5 \mathrm{kV})$.

forced air cooling is sufficient for practical applications of the ferroelectric lens even with the present loss of 2-2.5 dB.

As discussed earlier, to minimize the dielectric loss for obtaining $360^{\circ}$ differential phase shift, one would like to obtain as high a tunability as possible. Since tunability is a linear function of the dc bias voltage, one would like to apply as high a bias voltage as possible. Therefore, during experiments, bias voltage was increased gradually until dielectric breakdown occurred. Since ferroelectrics are good insulators, the dc current requirements are very low. For example, at $10-\mathrm{kV}$ bias voltage, the dc current drawn was $0.05 \mathrm{~mA}$ and so the dc power dissipated is only $0.5 \mathrm{~W}$. Fig. 13 shows the measured phase shift as a function of the bias voltage for various frequencies. As expected, the phase shift increases linearly with frequency because the electrical length of the material increases with frequency.

As dc bias voltage is applied to the ferroelectrics, the dielectric constant decreases, which is the basis for obtaining the differential phase shift, but it also complicates the impedance matching problem. However, our design optimization is able to cope with this difficult problem. Fig. 14 shows the reflection coefficients as a function of frequency for various bias voltages. The standing wave ratio (SWR) is less than two for a frequency range of $8-12 \mathrm{GHz}$, as theoretically predicted earlier.

It should be noted that considerable experimentation was performed to gain experience with high dielectric constant material loaded waveguide. Air gaps between the ferroelectric and the parallel conducting plates cause considerable problems with resonances. Poor electrodes will cause the electric field to concentrate in the air gaps causing breakdown; also, the field in the ferroelectric is reduced which decreases the ferroelectric tunability.

The experimental results presented here agree well with the theoretical results of the lens aperture matching. Control of the column phase shift with the applied dc voltage was also demonstrated experimentally. In summary, the experimental results on one column of the lens proved that the ferroelectric lens concept is viable and sound. Work is continuing in building and demonstrating a small lens.

\section{CONCLUSION}

A new concept for low-cost phased arrays using a voltagecontrolled ferroelectric lens was proposed. The ferroelectric lens phased array uniquely incorporates bulk phase shifting (the array does not contain discrete phase shifters) using ferroelectric material, which reduces significantly the number of phase shifters and drivers. Furthermore, row-column beam steering is used. These features, in turn, reduce the cost of the phased array. This paper presented the ferroelectric lens concept, its theoretical analysis, design, and experimental results. The theoretical results agree well with experimental results proving that the ferroelectric lens concept is very practical. It was shown that the lens surface can be matched very well to free-space over a broad range of frequencies and large scan angles. A number of low-cost phased-array configurations using the ferroelectric lens were also described.

\section{ACKNOWLEDGMENT}

The authors would like to thank Dr. L. Sengupta, Paratek Microwave Inc. (formerly of the Army Research Laboratory, Aberdeen Proving Ground, MD), for her contribution to the section on ferroelectric materials. They would also like to thank A. Moffat and J. Valenzi for their help with the experimental work.

\section{REFERENCES}

[1] V. K. Varadan, D. K. Ghodgaonkar, V. V. Varadan, J. F. Kelly, and P. Gilkerdas, "Ceramic phase shifters for electronically steerable antenna systems," Microwave J., vol. 35, pp. 116-127, Jan. 1992.

[2] R. W. Babbitt, T. E. Koscica, and W. C. Drach, "Planar microwave electro-optic phase shifters," Microwave J., vol. 35, pp. 63-79, June 1992.

[3] R. H. Park, "Radant lens: Alternative to expensive phased arrays," Microwave J., vol. 24, pp. 101-105, Sept. 1981.

[4] J. Leibinger, "Demonstration of two axis electronic scanning by a hybrid radant-TM lens array," Final Tech. Rep. RL-TR-91-201, Rome Laboratory, Hanscom AFB, MA, Sept. 1991.

[5] S. B. Cohn, "Lens-type radiators," in Antenna Engineering Handbook, H. Jasik, Ed. New York: McGraw-Hill, 1961, ch. 14.

[6] E. Ngo, S. Stowell, L. C. Sengupta, M. E. O'Day, and R. Lancto, "Fabrication and characterization of barium strontium titanate and nonferroelectric oxide composites," in Proc. Mat. Res. Soc. Symp., Boston, MA, Nov. 1994, vol. 360, pp. 45-50.

[7] L. C. Sengupta, S. Stowell, E. Ngo, M. E. O'Day, and R. Lancto, "Barium strontium titanate and nonferroelectric oxide ceramic composites for use in phased array antennas," Integrated Ferroelect., vol. 8, pp. 77-88, 1995.

[8] J. B. L. Rao, D. P. Patel, L. C. Sengupta, J. Synowczynski, L. H. Chiu, E. Ngo, and S. Sengupta, "Ferroelectric materials for phased 
array applications," in Dig. IEEE Antennas Propagat. Soc. Int. Symp., Montreal, Canada, July 1997, pp. 2284-2287.

[9] J. B. L. Rao, "Low cost phased arrays," Rep. NRL/MR/5317-95-7793, Naval Res. Lab., Washington, DC, Nov. 1995.

[10] J. B. L. Rao and D. P. Patel, "Voltage controlled ferroelectric lens phased arrays," in Dig. IEEE Antennas Propagat. Soc. Int. Symp., Baltimore, MD, July 1996, pp. 1624-1627.

[11] J. B. L. Rao, G. V. Trunk, and D. P. Patel, "Two low-cost phased arrays," in Proc. IEEE Int. Symp. Phased Array Syst. Technol., Boston, MA, Oct. 1996, pp. 119-124.

[12] J. B. L. Rao, D. P. Patel, and V. Krichevsky, "Voltage controlled ferroelectric lens phased arrays," Naval Res. Lab., Washington, DC, Rep. NRL/FR/5310-98-9883, Sept. 1998.

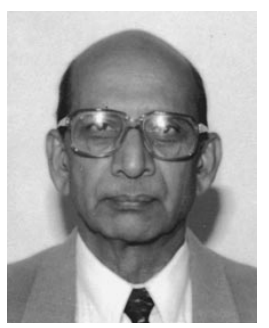

Jaganmohan B. L. Rao (S'65-M'66-SM'78) received the B.Sc. degree in physics from Andhra University, India, in 1956, the postgraduate diploma (D.M.I.T.) in electronics from the Madras Institute of Technology, India, in 1959, and the M.S. and $\mathrm{Ph} . \mathrm{D}$. degrees in electrical engineering from the University of Washington, Seattle, in 1963 and 1966, respectively.

Since 1974, he has been with the Radar Division of the Naval Research Laboratory, Washington, DC. $\mathrm{He}$ is currently the Head of the Antenna Section of the Radar Analysis Branch. He has published over 90 NRL reports and journal articles in antennas, electromagnetics, and related areas. He is a coauthor of "Microwave ferroelectric devices," in Encyclopedia of Electrical and Electronics Engineering (J. G. Webster, Ed. New York: Wiley, 1999).

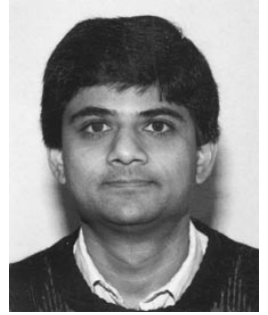

Dharmesh P. Patel (S'86-M'87) received the B.S degree in electrical engineering from Pennsylvania State University, University Park, PA, in 1987, and the M.S. degree in electrical engineering from the Johns Hopkins University, Baltimore, MD, in 1991.

From 1988 to 1991, he was with the Naval Surface Warfare Center, Silver Spring, MD. Since 1991 he has been with the Radar Division of the Naval Research Laboratory, Washington, DC. Currently, he is in the Antenna Section of the Radar Analysis Branch. His research interests include phased-array antennas and applications of ferroelectric materials at microwave frequencies. $\mathrm{He}$ is the author of "Microwave ferroelectric devices," in Encyclopedia of Electrical and Electronics Engineering (J. G. Webster, Ed. New York: Wiley, 1999).

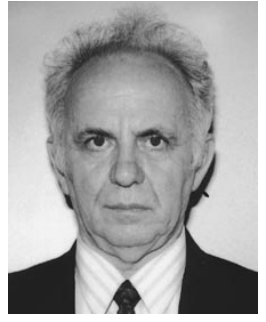

Vladimir Krichevsky (M'77) received the M.S. degree from Leningrad University, Russia, and the Ph.D. degree from Kharkov University, Ukraine, 1961 and 1971, respectively.

He is currently with the Naval Research Laboratory, Washington, DC. Previously, he was with MITRE Corporation, McLean, VA, COMSAT Laboratories, Clarksburg, MD, as well as the Electromagnetics Laboratory, University of Illinois, Urbana His professional interests include antenna development, electromagnetic wave propagation and scat- 